\title{
Mass Analysis of Persuccinated Derivatives of Neutral Oligosaccharides Using Matrix-Assisted Laser Desorption/Ionization Time-of-Flight Mass Spectrometry
}

\author{
Yeong Hee AHN*†, Jong Shin YoO* and Sung Ho KIM** \\ * Mass Spectrometry Group, Korea Basic Science Institute, Taejon 305-333, Korea \\ **Department of Chemistry, Sooncheonhyang University, Asan 337-880, Korea
}

\begin{abstract}
Matrix-assisted laser desorption/ionization time-of-flight mass spectrometry (MALDI-TOF MS) was used for the mass determination of the persuccinated derivatives of neutral oligosaccharides. The persuccinated oligosaccharides were derivatized by reacting neutral oligosaccharides with succinic anhydride in pyridine. The analytes were detected mainly as $(\mathrm{M}+\mathrm{Na})^{+}$ions with $100 \mathrm{fmol}$ sensitivity. This method is $10^{5}$ times more sensitive than the direct detection of underivatized oligosaccharides. This new persuccination method can be applied not only to linear oligosaccharides but also to cyclic oligosaccharides which do not have a free hydroxy group at the anomeric site like cyclodextrins. No inorganic reagent is used and no solvent extraction or purification procedure is necessary. So this method is very convenient and efficient for the highly sensitive detection of a variety of oligosaccharides.
\end{abstract}

Keywords Matrix-assisted laser desorption/ionization time-of-flight mass spectrometry, oligosaccharide, persuccination, succinic anhydride

It is well recognized that oligosaccharides have a variety of biologically important roles both as free carbohydrates and as constituents of glycoconjugates. In plants, free oligosaccharides play important roles in carbon fluxes within the cells and as regulators. ${ }^{1-3}$ Oligosaccharides covalently bound to proteins also influence protein stability, folding and several biological functions of glycoprotein itself. ${ }^{4-6}$ An intercellular recognition by protein may be affected by the structure and nature of the oligosaccharides. ${ }^{7}$ Thus the chracterization of complex oligosaccharides obtained from a variety of biological media has become of increased importance and has been conducted using a variety of analytical techniques.

Mass spectrometry has been recognized to be a highly useful technique for obtaining molecular weights of a variety of oligosaccharides and glycoconjugates. Especially, matrix-assisted laser desorption/ionization time-of-flight mass spectrometry (MALDI-TOF MS) has been used for the molecular weight determination of compounds having high molecular weight such as oligosacchrides, proteins and synthetic polymers. ${ }^{8-12}$ Unfortunately, due to the low proton affinity of oligosaccharides, the sensitivity and the detectable mass range of oligosaccharides, especially neutral oligosaccharides, are low and narrow compared with those of proteins. Thus in order to improve the sensitivity and the detectable mass range of oligosaccharides, several methods such as permethylation,

To whom correspondence should be addressed. peracetylation and reductive amination have been introduced. ${ }^{13-16}$ But in the analyses of derivatized oligosaccharides by these methods some purification procedures such as solvent extraction and chromatographic separation are necessary. These procedures are sometimes laborious and can be severely problematic, especially when only a small amount of the sample is available. The reductive amination method, forming a covalent bond with a variety of ligands containing nitrogen atom, will not be applicable to the oligosaccharides without a free hydroxy group at the glycosyl donor site (anomeric carbon) as in cyclodextrins.

Thus for the convenient and highly sensitive detection of a variety of oligosaccharides including oligosaccharides without a free hydroxy group at the glycosyl donor site, we have developed a new persuccination method. We chose $\alpha$-cyclodextrin, $\beta$-cyclodextrin, $\gamma$ cyclodextrin and maltoheptaose as model oligosaccharides (Fig. 1) and prepared the persuccinated oligosaccharide by the reaction of each oligosaccharide with succinic anhydride, which were analyzed by MALDITOF MS

\section{Experimental}

\section{Reagents}

Dimethyl formamide and pyridine were used after purification by the standard methods. ${ }^{17}$ After standing with anhydrous magnesium sulfate for $24 \mathrm{~h}$, dimethyl formamide was filtered and distilled under reduced 
a)

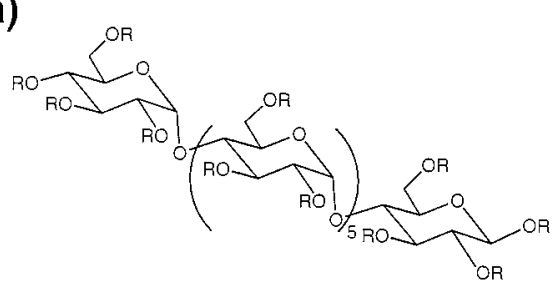

b)

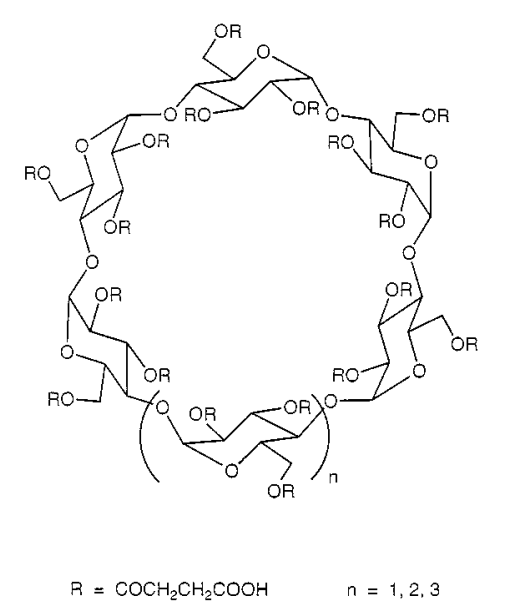

Fig. 1 Structures of persuccinated maltoheptaose (a) and cyclic oligosaccharides (b).

pressure. Pyridine was distilled after refluxing with potassium hydroxide pellets. Succinic anhydride, $N, N$ dimethylaminopyridine, $\alpha$-cyclodextrin, $\beta$-cyclodextrin, $\gamma$-cyclodextrin, maltoheptaose and 2,5-dihydroxybenzoic acid were purchased from Sigma Co.

\section{Derivatization with succinic anhydride}

Maltoheptaose $(1.15 \mathrm{mg}, 1 \mu \mathrm{mol})$ and succinic anhydride (46 mg, $0.46 \mathrm{mmol}, 20$ eq.) were dissolved in dry pyridine $(0.5 \mathrm{ml}) . \quad N, N$-Dimethylaminopyridine $(0.6$ $\mathrm{mg}, 5 \mu \mathrm{mol})$ was added to the solution and the reaction mixture was stirred at $80-100^{\circ} \mathrm{C}$ for $18 \mathrm{~h}$. $\alpha$-Cyclodextrin, $\beta$-cyclodextrin, $\gamma$-cyclodextrin and maltoheptaose were also derivatized in one tube to their persuccinates by the same method, except for a longer reaction time (about $24 \mathrm{~h}$ ).

\section{Preparation of sample}

A portion of the reaction mixture $(5 \mu \mathrm{l})$ was taken up and pyridine was removed under high vacuum. The residual analyte was dissolved in distilled water (100 $\mu \mathrm{l})$. One microliter of the aqueous solution was taken up and diluted with water/ethanol $(4 \mu \mathrm{l} / 3 \mu \mathrm{l})$. To this analyte solution $2 \mu \mathrm{l}$ of the separately prepared matrix solution was added. The matrix solution was prepared by dissolving $10 \mathrm{mg}$ of 2,5-dihydroxybenzoic acid in water/ethanol $(50 \mu \mathrm{l} / 50 \mu \mathrm{l})$. The analyte matrix mixture $(0.1 \mu \mathrm{l})$ was loaded on a stainless-steel probe and the solvent was evaporated under reduced pressure.

\section{MALDI-TOF mass spectrometer}

All MALDI-TOF mass spectrometry experiments were conducted using a Kompact MALDI II mass spectrometer (Kratos Company, Manchester, UK). The spectrometer consisted of a nitrogen laser with $5 \mathrm{~ns}$ pulse width at $337 \mathrm{~nm}$.

The laser power was attenuated to about $70 \%$ of the full power for optimum peak intensity. The ion accelerating voltage was set at $20 \mathrm{kV}$. The laser was focused onto the sample spot of about $30 \mu \mathrm{m}$ diameter. Mass spectra were typically accumulated from 30 laser shots.

\section{Results and Discussion}

After maltoheptaose was reacted with succinic anhydride for $4 \mathrm{~h}$, MALDI-TOF mass spectrometric analysis was carried out with a portion of the analyte taken up from the reaction mixture. Figure 2 a shows the MALDI-TOF MS spectrum obtained for 1 pmol of the partially succinated maltoheptaose derivative. Generally, the peaks due to pyridine and $N, N$-dimethylaminopyridine were observed at $\mathrm{m} / \mathrm{z} 80$ and 123 , respectively, in the low molecular weight region. The signals due to the matrix used were observed at $\mathrm{m} / \mathrm{z} 155$ and 137. In the high molecular weight region of the spectrum in Fig. 2a, several peaks due to partially succinated derivatives of maltoheptaose appeared at $\mathrm{m} / \mathrm{z}$ 2976, 3080 and 3175 with a same mass interval $(\Delta m / z=100)$, which was the mass difference expected for each additional succinate group. Among these peaks, the signal shown at $\mathrm{m} / \mathrm{z} 3080$ corresponded to the sodium adduct ion (calculated value, $\mathrm{m} / \mathrm{z}$ 3077) of

a)

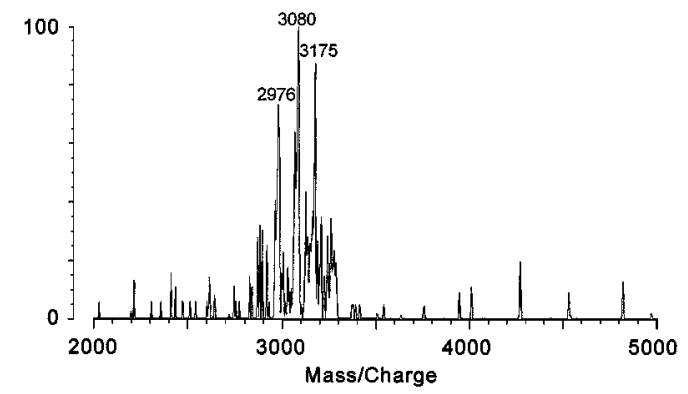

b)

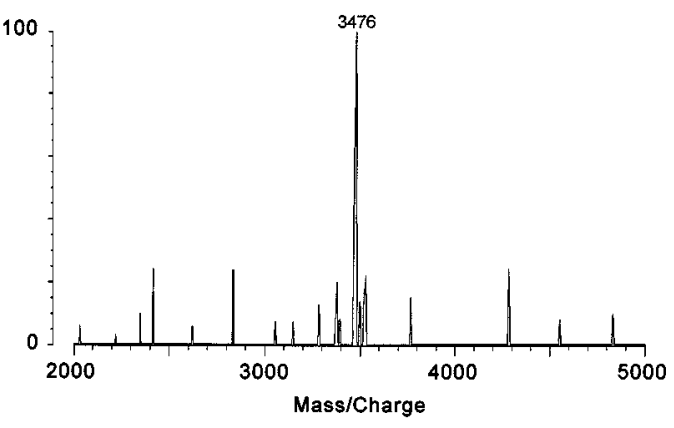

Fig. 2 MALDI-TOF MS spectra obtained for 1 pmol of maltoheptaose derivatives reacted for $4 \mathrm{~h} \mathrm{(a)}$ and for $18 \mathrm{~h}$ (b). 
$\mathrm{M}_{\text {malto }}+\mathrm{Suc}_{19}$. So we could diagnose the extent of the reaction using this method. Figure $2 \mathrm{~b}$ shows the MALDI-TOF MS spectrum obtained for 1 pmol of the completely succinated maltoheptaose derivative after maltoheptaose reacted with succinic anhydride for $18 \mathrm{~h}$. In order to increase the reaction rate, we used $N, N$ dimethylaminopyridine as a hypernucleophilic acylation catalyst. The peak due to the sodium adduction (calculated value, $\mathrm{m} / \mathrm{z}$ 3477) of the completely succinated maltoheptaose, $\mathbf{M}_{\text {malto }}+\mathrm{Suc}_{23}$, was observed at $\mathrm{m} / \mathrm{z}$ 3476. There was no noteworthy signal for the fragmented moiety. Thus we could detect the derivative of maltoheptaose with the signal-to-noise ratio, $S / N=4$, at 1 pmol level.

Cyclic oligosaccharides like cyclodextrin needed a longer reaction time (about $24 \mathrm{~h}$ ) for the complete persuccination than maltoheptaose. The slower reaction is probably due to the increased steric hindrance of the congested hydroxy groups in cyclic oligosaccharide than in maltoheptaose. Figures $3 \mathrm{a}$ and $3 \mathrm{~b}$ show the MALDI-TOF MS spectra obtained for 1 pmol of persuccinated $\alpha$-cyclodextrin derivatives after reaction for 4 and 24 h, respectively. Figure 3 a shows the mass difference $(\Delta m / z \fallingdotseq 100)$ due to each additional succinate group. The signal at $m / z 2396$ corresponds to the sodium adduct ion (calculated value, $m / z$ 2397) of the partially persuccinated derivative of $\alpha$-cyclodextrin, $\mathrm{M}_{\alpha \text {-CD }}$ + Suc $_{14}$. The peak of the sodium adduct (calculated value, $m / z 2797$ ) of completely persuccinated $\alpha$ cyclodextrin, $\mathbf{M}_{\alpha-\mathrm{CD}}+\mathrm{Suc}_{18}$, appeared at $m / z 2797$ in Fig. $3 b$.

In order to check the applicability of this persuccination method to the analytes in the mixture of several

a)

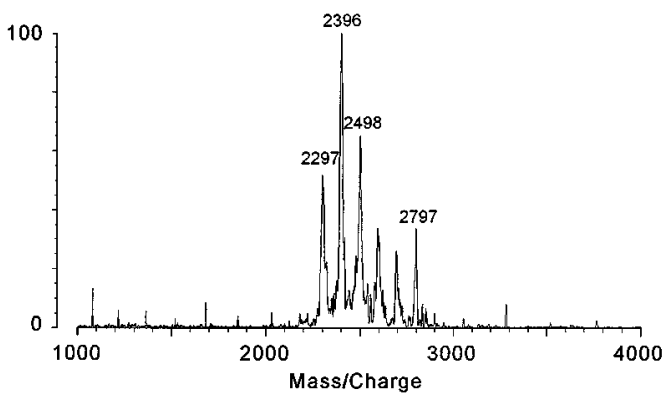

b)

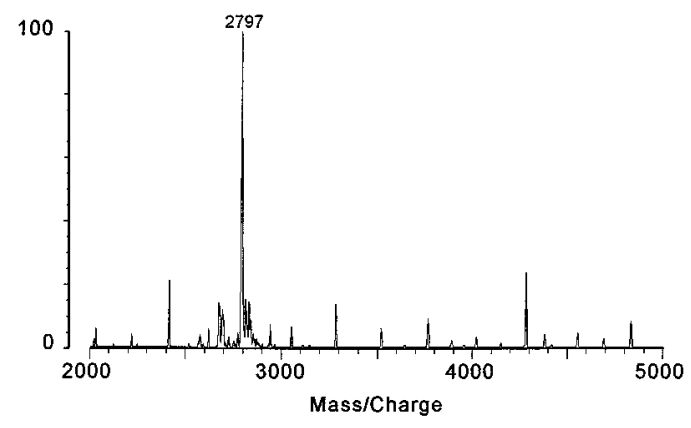

Fig. 3 MALDI-TOF MS spectra obtained for 1 pmol of $\alpha$ cyclodextrin derivatives reacted for $4 \mathrm{~h}$ (a) and for $24 \mathrm{~h}(\mathrm{~b})$. a)

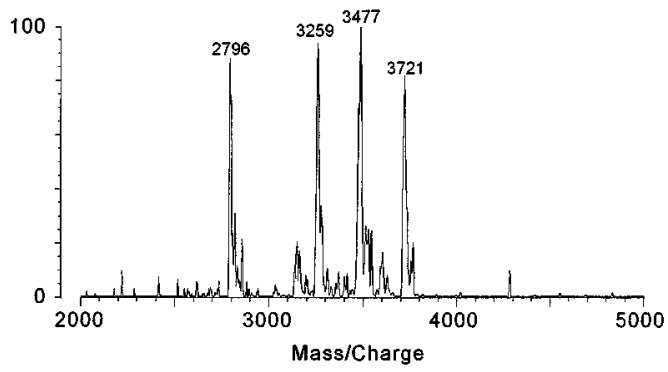

b)

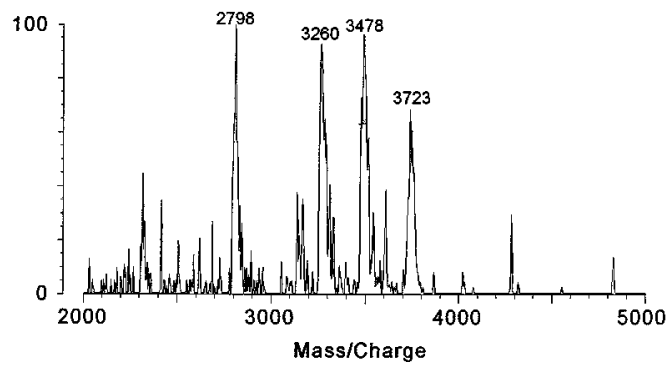

c)

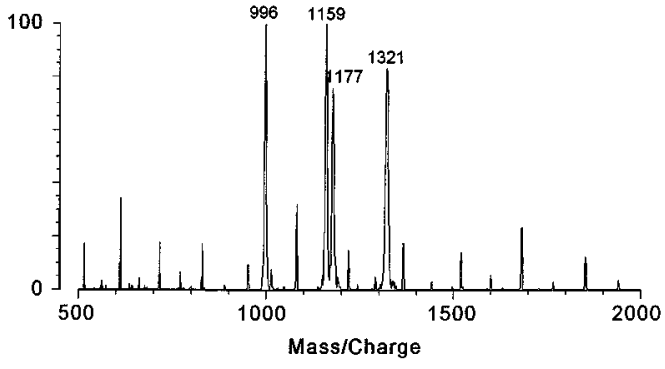

Fig. 4 MALDI-TOF MS spectra obtained for the analyte mixture: (a) obtained for $1 \mathrm{pmol}$ and (b) obtained for $100 \mathrm{fmol}$ of each persuccinated derivative of $\alpha$-cyclodextrin, $\beta$-cyclodextrin, maltoheptaose and $\gamma$-cyclodextrin; (c) obtained for 10 nmol of each free $\alpha$-cyclodextrin, $\beta$-cyclodextrin, maltoheptaose and $\gamma$-cyclodextrin.

kinds of oligosaccharides, we conducted the MALDITOF mass spectrometric analysis for a mixture of $\alpha$ cyclodextrin, $\beta$-cyclodextrin, $\gamma$-cyclodextrin and maltoheptaose. The persuccination of oligosaccharide mixture was also completed in $24 \mathrm{~h}$, as in the case of each pure oligosaccharide. Figure $4 \mathrm{a}$ shows the MALDITOF MS spectrum obtained for the analyte mixture which is composed of 1 pmol of each oligosaccharide. The signals of each sodium adduct ion (calculated value respectively, $m / z$ 2797, 3259, 3477 and 3721) formed from the persuccinated derivatives of $\alpha$ cyclodextrin, $\beta$-cyclodextrin, maltoheptaose and $\gamma$ cyclodextrin appeared at $\mathrm{m} / \mathrm{z}, 2796,3259,3477$ and 3721 , respectively, with similar peak intensity. The spectrum showed a satisfactory signal-to-noise ratio at 1 pmol level. This sensitivity is comparable to that for each pure oligosaccharide analyte. Furthermore with a signal-to-noise ratio, $S / N=2$, these derivatized oligosaccharides could be detected at $100 \mathrm{fmol}$ level (Fig. 4b). This sensitivity of persuccinated oligosaccharides is $10^{5}$ times better than that of underivatized free oligosaccharides obtained under the same analytical conditions in 
our laboratory. A mixture of free oligosaccharides was detected at $10 \mathrm{nmol}$ level with a similar signal-to-noise ratio, $S / N=2$, on the same mass spectrometer (Fig. 4c).

The sensitivity of persuccinated oligosaccharide is comparable with that of oligosaccharides derivatized by the reductive amination method. ${ }^{14-16}$ But since no inorganic reagent such as sodium hydride or sodium cyanoborohydride is used in this persuccination reaction and solvent extraction and purification procedures are not necessary, this new method is very convenient and efficient for the highly sensitive detection (100 fmol level) of a variety of oligosaccharides including oligosaccharides, without a free hydroxy group at the glycosyl donor site.

\section{References}

1. O. Kandler and H. Hopf, "The Biochemistry of Plants", ed. J. Preiss, Vol. 3, p. 221, Academic Press, New York, 1980.

2. M. Steup, "The Biochemistry of Plants", ed. J. Preiss, Vol. 14, p. 255, Academic Press, New York, 1988.

3. K. Tran, P. Toubart, A. Cousson, A. G. Darvill and D. J. Gollin, Nature [London], 314, 615 (1985).

4. A. Varki, Glycobiology, 3, 97 (1993).

5. R. A. Dwek, Chem. Rev., 96, 683 (1996).

6. O. P. Bahl, “Glycoconjugate: Composition, Structure and
Function", ed. H. J. Allen and E. C. Kisailus, p. 1, Dekker, New York, 1992.

7. E. Bollensen and M. Schachner, Neurosci. Lett., 82, 77 (1987).

8. B. Stahl, M. Steup, M. Karas and F. Hillenkamp, Anal. Chem., 63, 1463 (1991)

9. D. Garozzo, E. Spina, L. Sturiale, G. Montaudo and R. Rizzo, Rapid Commun. Mass Spectrom., 8, 358 (1994).

10. M. T. Cancilla, S. G. Penn and C. B. Lebrilla, Anal. Chem., 70, 663 (1998)

11. R. C. Beavis and B. T. Chait, Rapid Commun. Mass Spectrom., 3, 436 (1989).

12. J. S. Kim and J. S. Yoo, Analytical Science \& Technology, 8, 465 (1995).

13. S. J. Hakomori, Biochem. [Tokyo], 55, 205 (1964).

14. G. Okafo, J. Langridge, S. North, A. Organ, A. West, M. Morris and P. Camilleri, Anal. Chem., 69, 4985 (1997).

15. H. Suzuki, O. Muller, A. Guttman and B. L. Karger, Anal. Chem., 69, 4554 (1997).

16. T. Takao, Y. Tambara, A. Nakamura, K. Yoshino, H. Fukuda, M. Fukuda and Y. Shimonishi, Rapid Commun. Mass Spectrom., 10, 637 (1996).

17. D. D. Perrin, W. L. F. Armarego and D. R. Perrin, "Purification of Laboratory Chemicals", Pergamon Press, Oxford, 1988.

(Received July 7, 1998) (Accepted October 14, 1998) 